\title{
La Alerta de Violencia de Género en el Estado de México. Una reflexión sobre su implementación desde la sociedad civil
}

\section{Yenifar Carina Gómez Madrid ${ }^{1}$}

RESUMEN: Con el objetivo de analizar las acciones de prevención, seguridad y justicia implementadas a cuatro años de la declaración de la Alerta de Violencia de Género en el Estado de México (AVGEM), se presentan los principales obstáculos que no han permitido su correcta implementación, lo que significa un área de oportunidad para las instituciones. Para ello, se utilizó la metodología documental, que consistió en revisar distintos documentos oficiales de la Comisión Nacional para Prevenir y Erradicar la Violencia contra las Mujeres (Conavim), decretos del Congreso Local, acuerdos del ejecutivo estatal e informes sombras de organizaciones de la sociedad civil peticionarias y de seguimiento de la AVGEM. Aunque los resultados no son favorables por la falta de indicadores de evaluación y seguimiento, una realidad es que al menos el Mecanismo de Seguimiento, al estar en la Ley de Acceso de las Mujeres a una Vida Libre de Violencia del Estado de México, obliga la

\footnotetext{
${ }^{1}$ Universidad Autónoma del Estado de México / Mujeres en Incidencia A.C., contacto: yenifar@hotmail.com
} 
Artículo - Derechos Humanos de las Mujeres

coordinación estatal y municipal de las instituciones pese a las resistencias políticas y culturales.

Palabras clave: Violencia contra las mujeres; feminicidio; alerta de género; políticas públicas.

Abstract: The objective of the article is to reflect on the operation and meaning that the responsible authorities have given to the Gender Violence Alert (AVG) in the State of Mexico, four years after its declaration. For this, the documentary methodology was used to review different official documents of the National Commission to Prevent and Eradicate Violence against Women (Conavim), Local Congress decrees, state executive agreements and shadow reports of petitioner civil society organizations and of monitoring of the AVG. Although the results are not favorable due to the lack of evaluation and monitoring indicators, one reality is that at least the mechanism achieved state and municipal coordination despite political and cultural resistance.

Keywords: Violence Against Women; Femicide; Gender alert, Public Policy.

\section{Introducción}

C

on el objetivo de analizar las acciones de prevención, seguridad y justicia implementadas a cuatro años de la declaratoria de Alerta de Violencia de Género en el Estado de México, se presentan los principales obstáculos que no han permitido su correcta implementación, lo que sigue siendo un área de oportunidad para mejorar la coordinación entre las instituciones estatales y municipales encargadas de atender, prevenir, sancionar y erradicar la violencia contra las mujeres.

Para ello, se utilizó la metodología documental que consistió en revisar documentos oficiales de la Comisión Nacional para Prevenir y Erradicar la Violencia contra las mujeres (Conavim), decretos del Congreso Local del Estado de México, acuerdos del ejecutivo estatal, informes de los municipios reportados al Mecanismo de Seguimiento e informes sombras de organizaciones de la sociedad civil peticionarias y de seguimiento de la Alerta de Violencia de Género contra las Mujeres (AVGEM).En esta lógica, el artículo está dividido en tres partes, en la primera se exponen las principales acciones estatales implementadas a partir de la declaratoria, en el segundo apartado se describe de manera general los resultados que 


\section{Artículo - Derechos Humanos de las Mujeres}

encontró la sociedad civil mexiquense respecto a las acciones municipales reportadas al Mecanismo de Seguimiento que establece la Ley de Acceso del Estado de México, y en el tercero los obstáculos institucionales que no han permitido su correcta implementación.

La Alerta de Violencia de Género contra las mujeres es un mecanismo único regulado en el Titulo Segundo, capítulo V de la Ley General de Acceso de las Mujeres a una Vida Libre de Violencia para garantizar la seguridad de las mujeres, a través del "conjunto de acciones gubernamentales de carácter emergente, para enfrentar y erradicar la violencia feminicida en un territorio determinado, ya sea ejercida por individuos o por la propia comunidad" (artículo 22, Ley General), producto de omisiones, vacíos legislativos y agravios comparados cometidos por autoridades responsables de prevenir, atender y sancionar las violencias contra las mujeres.

El Título Tercero, capítulo I de su Reglamento establece el proceso de solicitud —por medio de organizaciones de la sociedad civil, organismos internacionales y de derechos humanos- y procedencia de la AVGEM, reformado en 2013 debido que en la sociedad civil recaían los medios de prueba y en el ejecutivo la procedencia, lo que generó un obstáculo para la emisión de las mismas y con la reforma se incorpora la participación de la Conavim y se establece la coordinación entre la Secretaria Ejecutiva (Inmujeres) y el Sistema Nacional de Prevención, Atención, Sanción y Erradicación de la Violencia contra las Mujeres. Además se adiciona en el artículo 36 la figura Grupo de Trabajo especificando quienes lo conforman y cuál es su función. Es por ello, que existen dos procedimientos para la declaratoria de alerta, "aquellos iniciados a partir de solicitudes formuladas con base en el Reglamento de 2008, y aquellas solicitudes presentadas luego de la reforma del 2013” (Ramírez, 2015).

En este sentido, el Observatorio Ciudadano Nacional del Feminicidio (OCNF) entre 2005 y 2010 registró 992 homicidios con características feminicidas, de los cuales sólo 18\% fueron investigados como tal. Para el periodo 2011-2013 registró 600 homicidios con estas características y más de 1,258 casos de desapariciones de mujeres (CMDPDH, 2015). Lo que motivó al OCNF${ }^{2}$, con base en el Reglamento de 2008, solicitar el 08 de diciembre de 2010 al Sistema Nacional la declaratoria para el Estado de México, a través de la Comisión Mexicana de Defensa y Promoción de los Derechos Humanos (CMDPDH).

\footnotetext{
${ }^{2}$ Solicitud de la AVG, disponible en https://www.gob.mx/cms/uploads/attachment/file/337607/Solicitud AVGM EdoMex 2010 OK.pdf
} 
Sin embargo, el 11 de enero de 2011 la solicitud de procedencia fue rechazada porque la investigación presentada por la organización fue considerada errónea y tras cuatro años de amparos por el retardo injustificado en la emisión de la declaratoria ${ }^{3}$ y campañas informativas para presionar al Sistema Nacional como "Ni un Feminicidio más en el Estado de México, Alerta de Violencia de Género, Ya" \#AVGYA realizada con colectivas del Estado de México y los resultados del Informe del Grupo de Trabajo fue posible en 2015 que el Sistema Nacional emitiera el 28 de julio la AVGEM para once municipios del Estado de México4: Chalco, Chimalhuacán, Cuautitlán Izcalli, Ecatepec, Ixtapaluca, Naucalpan de Juárez, Nezahualcóyotl, Tlalnepantla de Baz, Toluca, Tultitlán y Valle de Chalco donde se concentró 54\% (490) de los feminicidios entre 2005 y 2010 (Informe del Grupo de Trabajo, 2014).

Días antes de la emisión de procedencia, en el marco de la firma del convenio de coordinación con el Inmujeres, el 5 de julio de 2015, el ex gobernador Eruviel Ávila expresó públicamente su intención de solicitar de manera oficial al Sistema Nacional la declaratoria para once municipios del estado. Su discurso dio a conocer que el mecanismo fue concebido como una herramienta para consolidar y fortalecer programas sociales, acciones de gobierno y políticas públicas para combatir la violencia contra las mujeres mostrando desconocimiento de la Ley General y su Reglamento. Una vez declarada la AVGEM por feminicidio empezó un arduo camino para las organizaciones peticionarias y de seguimiento que han estado insistiendo y observando que las acciones de prevención, seguridad y justicia se lleven a cabo pero las tensiones políticas, desconocimiento del tema, falta de presupuesto, ensayos y errores y la propia dinámica y cultura institucional de la administración pública estatal y municipal ha obstaculizado su correcta implementación.

\section{De la acción ciudadana a los hechos institucionales}

En diciembre de 2015, entró en vigor el Decreto del ejecutivo para atender la Declaratoria de Alerta de Violencia de Género contra las mujeres para el Estado de México ${ }^{5}$, en cumplimiento al cuarto resolutivo de las medidas de seguridad que establece la declaratoria. En 2016 el

\footnotetext{
${ }^{3}$ Dictamen sobre la solicitud de la AVG, disponible en https://www.gob.mx/cms/uploads/attachment/file/337579/Dictamen sobre la solicitud de AVGM.pdf

${ }^{4}$ Declaratoria de Procedencia, disponible en https://www.gob.mx/cms/uploads/attachment/file/63107/DECLARATORIA ESTADO DE MEXICO.pdf

${ }^{5}$ Disponible para consulta

http://alertadegenero.edomex.gob.mx/sites/alertadegenero.edomex.gob.mx/files/files/principales/Decreto Des ap\%20(2).pdf
} 
gobierno del estado se vio presionado por la sociedad civil y los medios de comunicación para encaminar las acciones derivadas de la AVGEM pero el desconocimiento del tema; los vacíos legales en la armonización de la Ley de Acceso de las Mujeres a una Vida Libre de Violencia del Estado de México, y la falta de voluntad política se vieron reflejados en la desarticulación de las acciones.

Fue hasta diciembre de 2016 que entra en vigor el Decreto 181 el cual establece en el Capítulo I TER, el Mecanismo de Seguimiento (MS) de las medidas de seguridad, prevención y justicia para atender y erradicar la violencia contra las niñas, adolescentes y mujeres en el Estado de México (artículo 36 Bis de la Ley de Acceso). Lo que significó un gran avance pues al quedar institucionalizado en la ley, se establece la coordinación entre el ejecutivo y los municipios, a través de la Consejería Jurídica, obligando a las dependencias estatales y municipales que conforman el mecanismo dar seguimiento a las acciones contenidas en la AVG. Para 2017, se concretaron diversas acciones derivado del Decreto Administrativo, por ejemplo, entró en vigor el Protocolo Alba y el Poder Ejecutivo emitió el Acuerdo por el que se establecieron los mecanismos para la operación de los 30 millones de pesos para la mitigación de la AVGEM a cargo de la Unidad de Administración del Fondo de Ayuda, Asistencia y Reparación Integral de la Comisión Ejecutiva de Atención a Víctimas del Estado de México (CEAVEM). En sus artículos 5 y 6 establecen la operación del recurso para el fortalecimiento de las acciones que llevan a cabo los municipios. Las acciones a elegir consistieron en:

\section{Para la Comisión Ejecutiva:}
a. Talleres.
b. Diplomados.
c. Certificaciones.
d. Foros Regionales.
e. Capacitación al primer Interviniente.
f. Difusión de la campaña "No dejemos que las arranquen de nuestra vida".
g. Difusión de las acciones implementadas en atención a la alerta.
h. Adquisición de equipo informático.
i. Adquisición de parque vehicular.
j. Difusión de cédulas de búsqueda de personas desaparecidas.
k. Terapia de BurnOut.
1. Difusión de cartillas de derechos. 


\section{Artículo - Derechos Humanos de las Mujeres}

\section{2. . Para los Municipios:}

a. Talleres.

b. Diplomados.

c. Certificaciones.

d. Foros Regionales.

e. Capacitación al primer Interviniente.

f. Difusión de la campaña "No dejemos que las arranquen de nuestra vida".

g. Difusión de las acciones implementadas en atención a la alerta (GEM, 2017).

Al respecto, algunos Ayuntamientos ingresaron proyectos de diplomados, certificaciones, capacitaciones, pero no obtuvieron respuesta por parte de la CEAVEM y no accedieron a al recurso. Con el sexenio por concluir, se dificultó el seguimiento de las acciones siguiendo la lógica de cambio de gobierno y las organizaciones peticionarias se enfrentaron de nueva cuenta a las tensiones políticas, cambios administrativos, de titulares y enlaces. Una vez instalada la actual administración, en septiembre de 2018 se reforman diversas disposiciones de la Ley Orgánica de la Administración Pública del Estado de México, específicamente en su artículo 19 donde se establece la modificación de la denominación de Consejería Jurídica a Secretaría de Justicia y Derechos Humanos. En febrero de 2019, el Ejecutivo emite el Acuerdo del Secretario de Justicia y Derechos Humanos por el que se establecen los mecanismos para la operación de recursos para la mitigación de la AVGEM en los once municipios de la entidad. Los lineamientos ahora establecen que la Unidad de Apoyo Administrativo de la CEAVEM es quien debe operar los recursos para las siguientes acciones:

1. Talleres.

2. Diplomados.

3. Certificaciones.

4. Capacitación al Primer Interviniente.

5. Difusión de Cédulas de Búsqueda.

6. Adquisición de vehículos para el fortalecimiento de las Células de Búsqueda y de la Policía de Género.

7. Equipamiento para atención de mujeres víctimas de violencia (GEM, 2018).

En este sentido, entre las medidas implementadas para atender y mitigar la AVG en el Estado de México se encuentran: 


\section{Artículo - Derechos Humanos de las Mujeres}

Tabla 1.

Medidas implementadas para atender y mitigar la AVG en el Estado de México

\begin{tabular}{|c|c|c|}
\hline Medidas de Prevención & Medidas de Seguridad & Medidas de Justicia \\
\hline $\begin{array}{l}\text { Campaña "No dejemos que } \\
\text { las arranquen de nuestra } \\
\text { vida”; } \\
\text { Programa único de } \\
\text { capacitación, sensibilización, } \\
\text { formación y } \\
\text { Profesionalización } \\
\text { en materia de derechos } \\
\text { humanos de las mujeres para } \\
\text { las y los servidores públicos; } \\
\text { Criterios para la prestación } \\
\text { de servicios de salud cuando } \\
\text { reciban casos de violencia } \\
\text { familiar, sexual y contra las } \\
\text { mujeres, } \quad \text { Nom-046-SsA2- } \\
\text { 2005; } \\
\text { Instalación de } 11 \text { Unidades de } \\
\text { Atención Inmediata } \\
\text { especializadas en los } 11 \\
\text { municipios por parte del } \\
\text { Consejo Estatal de la Mujer y } \\
\text { Bienestar Social (CEMyBs); } \\
\text { víctimas"; } \\
\text { Creación de las Unidades de } \\
\text { Género; violencia de } \\
\text { Creación del Programa Único }\end{array}$ & $\begin{array}{l}\text { Aplicación del Protocolo de } \\
\text { Actuación Policial en materia } \\
\text { de violencia contra las } \\
\text { mujeres; } \\
\text { Georreferenciación de zonas } \\
\text { con mayor incidencia de } \\
\text { violencia contra las mujeres; } \\
\text { Acuerdo Interinstitucional } \\
\text { por el que se da a conocer el } \\
\text { Protocolo Alba Estado de } \\
\text { México; } \\
\text { Actualización del Banco de } \\
\text { Datos (BADEMVIM) dedicada } \\
\text { al registro de los casos de } \\
\text { violencia contra las mujeres. }\end{array}$ & $\begin{array}{l}\text { Creación de la Fiscalía } \\
\text { Central para la Atención de } \\
\text { Delitos Vinculados a la } \\
\text { Violencia de Género; Manual } \\
\text { y Protocolo de Atención a } \\
\text { Víctimas u ofendidos del } \\
\text { delito de feminicidio; } \\
\text { Puesta en marcha de Centros } \\
\text { de Justicia para las Mujeres: } \\
\text { Amecameca, Toluca, } \\
\text { Ecatepec y Cuautitlán Izcalli. } \\
\text { Unidad de Análisis y } \\
\text { Contexto para } \\
\text { Investigación de Delitos } \\
\text { Vinculados al Feminicidio; } \\
\text { Cambio de denominación por } \\
\text { el que se crea la Fiscalía } \\
\text { Especializada para la } \\
\text { Investigación y Persecución } \\
\text { de Delitos en Materia de } \\
\text { Desaparición Forzada de } \\
\text { Personas y Desaparición } \\
\text { Cometida por Particulares. }\end{array}$ \\
\hline
\end{tabular}




\section{Artículo - Derechos Humanos de las Mujeres}

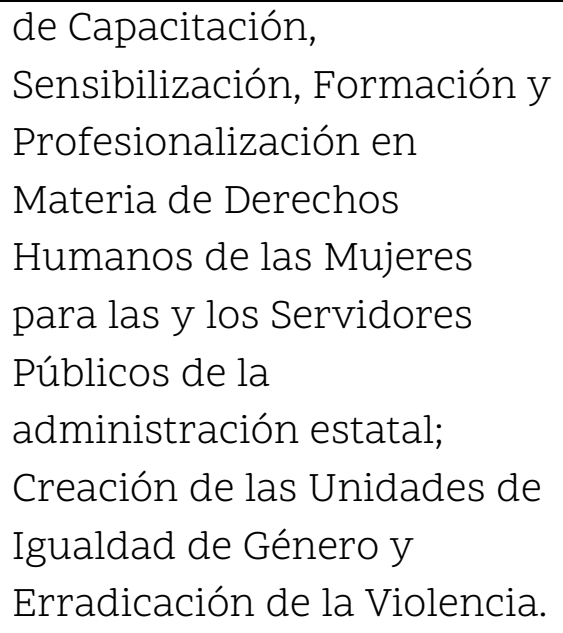

Fuente: elaboración propia con base en los acuerdos de sesión del GIM encargado de dar seguimiento a la AVG en el Estado de México 2015-2017 y documentos oficiales.

\section{Acciones locales y avances sin seguimiento}

A partir de los informes entregados por los municipios alertados al MS que establece la Ley de Acceso estatal, entre 2016 y 2017, la Comisión Estatal de Derechos Humanos del Estado de México (CODHEM) realizó con base en los lineamientos de la AVGEM un checklist de las acciones cumplidas o no cumplidas por parte de los ayuntamientos y en coordinación con el Observatorio Ciudadano en contra de la Violencia, Desaparición y Feminicidios en el Estado de México (OCMEXFEM) llevamos a cabo visitas in situ imprevistas a instituciones facultadas en la atención y seguridad de las mujeres víctimas de violencia de género, así como colonias georreferenciadas con mayor incidencia de violencia de género con el objetivo de corroborar la efectiva implementación de la AVGEM, toda la información recabada permitió a la Codhem presentar las áreas de oportunidad, recomendaciones y observaciones del ocmexfem ante el MS para mejorar el funcionamiento de la misma. Toda la información reportada se encuentra en el Informe Ejecutivo de Acciones en Atención a la Alerta de Violencia de Género contra las Mujeres en 11 Municipios del Estado de México (2018) y en el sitio web: Alerta de Género ${ }^{6}$ que antes pertenecía al Consejo Estatal de la Mujer y Bienestar Social (CEMyBS) y con las modificaciones legales y administrativas ahora está a

\footnotetext{
${ }^{6}$ Sitio web oficial de la Alerta de Género del Estado de México, disponible en http://alertadegenero.edomex.gob.mx/. Cabe mencionar que hasta la fecha las administraciones municipales actuales no han reportado acciones en el sitio.
} 
cargo de la Secretaría de Justicia y Derechos Humanos. Entre las acciones reportadas se encontraron:

\begin{tabular}{|c|c|c|c|}
\hline \multicolumn{4}{|l|}{ Tabla 2.} \\
\hline Municipio & Prevención & Seguridad & Justicia \\
\hline Chalco & $\begin{array}{l}\text { Rehabilitación e instalación de } \\
\text { alumbrado público; difusión de } \\
\text { números de emergencia en línea y } \\
\text { en calles. }\end{array}$ & $\begin{array}{l}\text { Patrullajes con } \\
\text { perspectiva de género; } \\
\text { búsqueda y localización } \\
\text { de personas. }\end{array}$ & $\begin{array}{l}\text { Medidas de } \\
\text { protección }\end{array}$ \\
\hline Chimalhuacán & $\begin{array}{l}\text { Talleres para prevenir la violencia } \\
\text { de género con mujeres de distintas } \\
\text { localidades y talleres para jóvenes } \\
\text { con el tema de micromachismos, } \\
\text { violencia en el noviazgo y } \\
\text { masculinidades; capacitación a } \\
\text { servidores y servidoras públicos. }\end{array}$ & & \\
\hline Cuautitlán Izcalli & $\begin{array}{l}\text { Difusión permanente de la AVG en } \\
\text { su micrositio } \\
\text { http://cuautitlanizcalli.gob.mx/imie } \\
\text { mh/; eventos culturales; campaña: } \\
\text { "No es de novios"; } \\
\text { Iluminación y rescate de espacios } \\
\text { públicos y pláticas para menores } \\
\text { sobre valores. }\end{array}$ & $\begin{array}{l}\text { Célula de Búsqueda de } \\
\text { personas desaparecidas }\end{array}$ & \\
\hline Ecatepec & $\begin{array}{l}\text { Aplicación inteligente "Alerta Rosa”; } \\
\text { empoderamiento de la mujer con la } \\
\text { rehabilitación de cuatro Casas de la } \\
\text { Mujer donde se brindan talleres } \\
\text { para el autoempleo; rescate de } \\
\text { espacios públicos; creación de la } \\
\text { Unidad Especializada de Atención a } \\
\text { Víctimas de Violencia Familiar y de } \\
\text { Género; pláticas y talleres en } \\
\text { escuelas de educación básica, } \\
\text { medio superior y superior en temas } \\
\text { de equidad de género, violencia en } \\
\text { el noviazgo. }\end{array}$ & $\begin{array}{l}\text { Célula de Búsqueda } \\
\text { Inmediata de Personas } \\
\text { Desaparecidas, No } \\
\text { Localizadas, Extraviadas } \\
\text { o Ausentes }\end{array}$ & \\
\hline
\end{tabular}


Artículo - Derechos Humanos de las Mujeres

\begin{tabular}{|c|c|c|c|}
\hline Municipio & Prevención & Seguridad & Justicia \\
\hline Ixtapaluca & $\begin{array}{l}\text { Operativo "El Vigilante", helicóptero } \\
\text { que recorre zonas } \\
\text { georreferenciadas; } \\
\text { empoderamiento económico de las } \\
\text { mujeres con talleres para el } \\
\text { autoempleo y conferencias en } \\
\text { escuelas de educación básica y } \\
\text { medio superior en temas de } \\
\text { violencia en el noviazgo, valores en } \\
\text { la familia, autoestima y emociones } \\
\text { tóxicas. }\end{array}$ & $\begin{array}{l}\text { Creación de la Policía de } \\
\text { Género }\end{array}$ & \\
\hline $\begin{array}{l}\text { Naucalpan de } \\
\text { Juárez }\end{array}$ & $\begin{array}{l}\text { Implementación del Modelo de } \\
\text { Atención a Medidas de Protección; } \\
\text { campaña "Prevengamos y } \\
\text { erradiquemos la violencia de } \\
\text { género"; capacitación a las y los } \\
\text { elementos de seguridad ciudadana. }\end{array}$ & $\begin{array}{l}\text { Célula de Recuperación de } \\
\text { Personas y Policía de } \\
\text { Género } \\
\text { Atención a mujeres en } \\
\text { situación de violencia; } \\
\text { implementación del } \\
\text { Modelo de Formación de } \\
\text { Enlaces Policiales; } \\
\text { operativo vigilancia y } \\
\text { seguridad en el } \\
\text { transporte público. }\end{array}$ & \\
\hline Nezahualcóyotl & $\begin{array}{l}\text { Rehabilitación Parque "Lago } \\
\text { Atitlán"; aplicación inteligente } \\
\text { "Neza Segura”; campaña: “Neza a } \\
\text { favor de la Igualdad". }\end{array}$ & $\begin{array}{l}\text { Policía de Género y Célula } \\
\text { de Búsqueda de Personas } \\
\text { Desaparecidas (Alerta } \\
\text { Neza); Policía Vecinal de } \\
\text { Proximidad y acciones de } \\
\text { infraestructura. }\end{array}$ & \\
\hline
\end{tabular}




\section{Artículo - Derechos Humanos de las Mujeres}

\begin{tabular}{|c|c|c|c|}
\hline MUNICIPIO & PREVENCIÓN & SEGURIDAD & JUSTICIA \\
\hline $\begin{array}{l}\text { Tlalnepantla de } \\
\text { Baz }\end{array}$ & $\begin{array}{l}\text { Jornadas de atención integral para } \\
\text { las Mujeres; Redes Comunitarias } \\
\text { (Modelo CEMyBs y Programa de } \\
\text { Fortalecimiento para la seguridad } \\
\text { (FORTASEG); pláticas en escuelas de } \\
\text { educación básica, media superior } \\
\text { sobre masculinidades, violencia en } \\
\text { el noviazgo, roles y estereotipos, } \\
\text { tipos y modalidades de la violencia } \\
\text { y derechos humanos de las } \\
\text { mujeres; campaña: "Tlalnepantla a } \\
\text { favor del respeto hacia la mujer" y } \\
\text { cine debates. }\end{array}$ & $\begin{array}{l}\text { Creación de la Policía de } \\
\text { Género y la Célula de } \\
\text { Búsqueda y Localización } \\
\text { de Mujeres y niñas } \\
\text { Desaparecidas }\end{array}$ & \\
\hline Toluca & $\begin{array}{l}\text { Creación de Redes Comunitarias } \\
\text { con base en el Modelo CEMyBS; } \\
\text { Recuperación de espacios públicos; } \\
\text { talleres en materia de sexualidad y } \\
\text { violencia de género; } \\
\text { empoderamiento de la mujer con } \\
\text { talleres para el autoempleo }\end{array}$ & $\begin{array}{l}\text { Creación de la Unidad } \\
\text { Especializada en Género; } \\
\text { capacitación a } \\
\text { servidores/as públicas }\end{array}$ & \\
\hline Tultitlán & $\begin{array}{l}\text { Redes Juveniles y Redes de Mujeres } \\
\text { contra la violencia; capacitación a } \\
\text { funcionariado municipal; pláticas } \\
\text { informativas y preventivas para la } \\
\text { juventud. }\end{array}$ & $\begin{array}{lcc}\text { Atención } & \text { integral } & \text { a } \\
\text { mujeres } & \text { víctimas } & \text { de } \\
\text { violencia } & & \end{array}$ & \\
\hline Valle de Chalco & \multicolumn{3}{|l|}{ No reportó acciones } \\
\hline
\end{tabular}

Fuente: elaboración propia con base en el portal oficial de la Alerta de Violencia de Género del Estado de México (2019)

\section{Obstáculos institucionales}

El problema en la implementación de la AVGEM ha sido la designación de enlaces que fungen como representantes de las y los titulares que establece la Ley de Acceso porque no tienen el perfil o conocimiento requerido para atender los lineamientos de la AVGEM y por su posición jerárquica al interior de la institución no pueden tomar decisiones para articular acciones, metas y tomar responsabilidades, que ha resultado en una falta de compromiso, 
Artículo - Derechos Humanos de las Mujeres

acciones desarticuladas, lo que repercute en el uso ineficiente de los recursos públicos y rendición de cuentas.

Un obstáculo ha sido también la lógica administrativa para ejercer los recursos públicos, ya que al ser por ejercicio fiscal puede haber subejercicio de recursos y no utilizarse como lo establece el Acuerdo Administrativo, aunado a que está dirigido a fortalecer las instituciones y no a la intervención directa en las localidades georreferenciadas con mayor incidencia de violencia contra las mujeres. Además, las autoridades no han atendido las recomendaciones del OCNF en cuanto a establecer indicadores de evaluación y seguimiento de las acciones de la AVGEM. Otro problema de las instituciones tanto estatales como municipales es que no está institucionalizada la perspectiva de género y mucho menos transversalizada, no cuentan con diagnósticos que permitan dimensionar las causas de las violencias y el feminicidio, lo que sigue siendo un área de oportunidad para las instituciones pero mientras tanto, las demandas ciudadanas exigen resultados concretos ante la ola de muertes violentas y desaparición de mujeres, niñas y adolescentes.

\section{Conclusiones}

Ante los cambios de gobierno estatal y municipal en 2017 y 2019, se observa la necesidad de implementar un mecanismo de evaluación y seguimiento para medir los avances y fortalecer las acciones de prevención, seguridad y justicia, ya que en la mayoría de los gobiernos municipales no le dieron continuidad a las acciones, por ejemplo, falta de conocimiento en el tema, cambio de partido político en la administración que gobierna, personal sin perfil. En los informes sombra de la sociedad civil resalta que los indicadores de proceso del gobierno del estado no obedecen a medir los resultados que respondan a las medidas de la AVGEM, por ejemplo, incorporar indicadores de impacto.

En cuanto a las acciones implementadas a nivel municipal, deja ver que la responsabilidad de atender la problemática recae principalmente en los Ayuntamientos, y si no se cuenta personal capacitado, sensible y presupuesto, no se cumplen las medidas solicitadas, aunado a que la Ley de Acceso de las Mujeres a una Vida Libre de Violencia del Estado de México no establece las acciones específicas que deben implementar los municipios, ya que se basan en la declaratoria de la AVGEM que en estos casos debería establecer una coordinación permanente para realizar ciertas acciones. Donde más incidencia se observa es por parte de la Fiscalía General del estado en cuanto a generar la Policía de Género, Preventiva y Búsqueda de personas, el problema es que no cuentan con recursos humanos suficientes, 
especializados/as en el tema, presupuesto etiquetado y operan con lo que tienen a su alcance. Respecto a las medidas de prevención, varias de las acciones estatales se homologan en los municipios alertados cuando cada localidad tiene sus particularidades y se corre el riesgo de no obtener el resultado esperado.

En materia de justicia es lo que más trabajo ha costado. Los complejos avances en las carpetas de investigación por feminicidio; la atención revictimizante de las y los Ministerios Públicos de algunos Centros de Justicia; la falta de perspectiva de género a la hora de juzgar sigue obstaculizando el acceso a una vida libre de violencia, la justicia y reparación del daño. Por ello, la AVGEM después de cuatro años no ha logrado garantizar la seguridad de las mujeres, responder a las demandas de la sociedad civil, atender de manera cabal las nueve conclusiones del Informe del Grupo de Trabajo, incluidas en las 18 acciones que establece la Declaratoria de Procedencia, ya que dependiendo el significado e importancia que le dan las autoridades estatales y municipales a la violencia contra las mujeres; la cultura organizacional en las instituciones obligadas a prevenir, atender y sancionar la violencia contra las mujeres sin perspectiva de género se ve reflejada en la operatividad de la AVGEM y muchas veces, aunque se tenga voluntad política de cambiar las cosas, mientras la cultura patriarcal atraviese las estructuras, la violencia contra las mujeres no será erradicada. 
Artículo - Derechos Humanos de las Mujeres

\section{Referencias}

Comisión Mexicana de Defensa y Promoción de los Derechos Humanos AC (2015), “Escrito inicial de demanda", Distrito Federal: CMDPDH.

Comisión Nacional de Derechos Humanos (2017), “Diagnóstico de la Comisión Nacional de los Derechos Humanos como integrante de los grupos de trabajo que dan seguimiento a los procedimientos de Alerta de Violencia de Género contralas Mujeres", Ciudad de México: CNDH.

Comisión Nacional para Prevenir y Erradicar la Violencia contra las Mujeres (2019), "Actas de sesión del Grupo Interinstitucional Multidisciplinario", consultadas en https://portales.segob.gob.mx/es/Transparencia/AVGM Estado de Mexico_ \20 de agosto de 2019].

Gobierno del Estado de México (2018), “Alerta de Género”, Toluca: Secretaría de Justicia y Derechos Humanos del Estado de México, consultada en http://alertadegenero.edomex.gob.mx/zonas riesgo [20 de agosto de 2019].

Gobierno del Estado (2019), “Acuerdo del Secretario de Justicia y Derechos Humanos por el que se establecen los mecanismos para la operación de recursos para la mitigación de la Alerta de Violencia de género contra las mujeres para el Estado de México en los once municipios de la entidad objeto de dicha alerta", Toluca: GEM.

Instituto Nacional de las Mujeres (2018), Informe de evaluación del funcionamiento del mecanismo, Ciudad de México: Inmujeres.

Poder legislativo (2016), “Decreto Número 181.- Por el que se reforman y adicionan diversas disposiciones de la Ley Orgánica de la Administración Pública del Estado de México a la Ley de Acceso de las Mujeres a una Vida Libre de Violencia del Estado de México", Toluca: Poder Legislativo.

Poder Legislativo del Estado Libre y Soberano (2007), “Ley General de Acceso de las Mujeres a una Vida Libre de Violencia”, México: Cámara de Diputados.

Poder Legislativo del Estado Libre y Soberano (2007), "Ley de Acceso de las Mujeres a una Vida Libre de Violencia del Estado de México”, Toluca: Poder Legislativo del Estado Libre y Soberano.

Poder Legislativo (2008), "Reglamento de la Ley General de Acceso de las Mujeres a una Vida Libre de Violencia”, México: Cámara de Diputados.

Poder Legislativo (2008), "Reglamento de la Ley General de Acceso de las Mujeres a una Vida Libre de Violencia, última reforma 14 de marzo de 2014”, Distrito Federal: Cámara de Diputados. 
Ramírez, Gloria (2018), "Informe Sombra sobre el Seguimiento de las recomendaciones del Comité de la CEDAW al Estado Mexicano 2018, Alertas de Género contra las Mujeres, Feminicidio, Desaparición, Norma 046", Ciudad de México: Cátedra Unesco de Derechos Humanos de la UNAM.

Secretaria de Justicia y Derechos Humanos del Estado de México (2018), "Informe Ejecutivo de Acciones en Atención a la Alerta de Violencia de Género contra las Mujeres en 11 Municipios del Estado de México", Toluca: Secretaria de Justicia y Derechos Humanos del Estado de México. 